RICYDE. Revista Internacional de Ciencias del Deporte doi: $10.5232 /$ ricyde

Rev. int. cienc. deporte

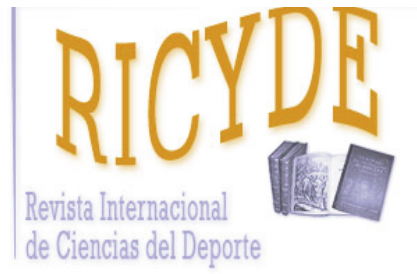

RICYDE. Revista Internacional de Ciencias del Deporte VOLUMEN XII - AÑO XII

Páginas:234-249 ISSN:1885-3137 Número 45 - Julio - 2016

\title{
Ampliando horizontes sobre medición del rendimiento y regularidad en el baloncesto profesional \\ Expanding horizons on performance measurement and regularity in professional basketball
}

\author{
Román Salmerón-Gómez, Samuel Gómez-Haro \\ Facultad Ciencias Económicas y Empresariales. Universidad de Granada
}

\begin{abstract}
Resumen
Conseguir información relevante sobre el rendimiento de equipos y jugadores para la toma de decisiones provoca un continuo análisis de factores que afectan al resultado individual y del conjunto en el ámbito del deporte. La necesidad de obtener resultados positivos hace necesaria el uso de herramientas de gestión que ayuden a la estimación fiable del rendimiento deportivo de los jugadores profesionales y así mejorar la toma de decisiones por los directivos deportivos, por ejemplo, en el momento de diseñar una plantilla. El presente trabajo trata de mejorar el análisis existente presentando un nuevo indicador para medir el rendimiento y la regularidad de los jugadores de baloncesto mostrando por qué es preferible a otros usados anteriormente en la bibliografía existente. El análisis empírico se centra en calcular la regularidad de todos los jugadores que hayan disputado al menos 10 partidos en la liga de la Asociación de Clubes de Baloncesto, conocida como Liga ACB, con una media de minutos jugados superior o igual a 10 durante la temporada 2014/2015. Los resultados obtenidos presentan perspectivas interesantes para aspectos de la gestión deportiva como el reclutamiento y diseño de equipos deportivos.
\end{abstract}

Palabras clave: rendimiento deportivo; regularidad; indicadores estadísticos; baloncesto profesional.

\begin{abstract}
Get relevant information about players and team performance to take decisions causes a continuous analysis of factors affecting individual and group performance in sport context. The need for positive results takes to use management tools that estimate athletic performance of professional players reliable in regards to the decision by the sport managers, for example, when they are designing the player roster. This study aims to improve the existing analysis by providing, on the one hand, new indexes to measure performance and regularity of professional basketball players, and showing how traditional statistics tools can detect relevant information. We use data from every player in terms of performance according to Spanish basketball league ACB in the 2014/2015 season with a minimum of ten games played and ten minutes averaged per game to redefine and show new elements to consider in assessing the performance of the players. The results obtained have interesting prospects for aspects of sports management as recruiting, roster designing and performance of sports teams.
\end{abstract}

Key words: sport performance; regularity; statistics indexes; professional basketball.

Correspondencia/correspondence: Samuel Gómez Haro

Facultad Ciencias Económicas y Empresariales. Departamento de Métodos Cuantitativos. Universidad de Granada. Email: samugh@ugr.es 
Salmerón-Gómez, R., y Gómez-Haro, S. (2016). Ampliando horizontes sobre medición del rendimiento y regularidad en el baloncesto profesional. RICYDE. Revista internacional de ciencias del deporte. 45(12), 234249. http://dx.doi.org/10.5232/ricyde2016.04502

\section{Introducción}

$\mathrm{D}$ esde el ámbito de la investigación, un objetivo dentro del amplio campo de la gestión deportiva ha sido descubrir herramientas que provean a los entrenadores y directivos de organizaciones de un conocimiento más profundo de los recursos que dispone de cara a mejorar su rendimiento. Esas funciones incluyen el diseño y la construcción de las plantillas, organización de los programas de entrenamiento, planificación de estrategias de temporada y de juego; y la relación de los jugadores-equipo con su rendimiento (Alamar, 2013; Drust, 2010). Esta información nos sirve para conocer las fortalezas y debilidades a nivel individual y de equipo, ya sea propio o del oponente con el que te enfrentas cada jornada, y el rendimiento en las diferentes situaciones existentes en un determinado contexto complejo como son los equipos deportivos profesionales. El análisis del rendimiento es un tema que siempre ha estado en continuo desarrollo, pero desde el fenómeno Moneyball (Lewis, 2004) vivimos en una continua revisión de los conceptos existentes y búsqueda de mejores técnicas de análisis de la información que ofrezca información valiosa para la toma de decisiones. En este trabajo desarrollamos nuevos instrumentos fiables que nos provean de una nueva visión del rendimiento individual de los jugadores en el juego colectivo dentro del contexto del deporte del baloncesto profesional, utilizando como ejemplo la liga española de baloncesto profesional, la liga de la Asociación de Clubes de Baloncesto-Liga ACB.

El baloncesto, como deporte de equipo, se caracteriza por la ejecución de una serie de habilidades en múltiples situaciones que surgen durante el desarrollo del juego. Con cada habilidad existe un amplio número de posibles soluciones que pueden ser tomadas, que dependen de las características de los jugadores, de su previa experiencia en el juego, etc. La mayoría de esas acciones tienen un determinado carácter (acciones ofensivas o defensivas, por ejemplo), así que las posibilidades de tener éxito son variadas (Gutiérrez y Ruiz, 2013).

Además, los deportes de equipo se ajustan dinámicamente acorde a las características de los equipos que disputan el partido y de los jugadores propios y del rival (Trninic', Trninic' y Papic', 2009). Para optimizar el resultado de un equipo es necesario considerar el grado de dominio del juego a nivel colectivo, el rendimiento de cada jugador, las relaciones con compañeros y oponentes. Así que tenemos un sistema complejo de variables que son imposibles de controlar en su totalidad. En el proceso de preparación de un equipo, los managers tratan de facilitar las condiciones para el desarrollo de las habilidades de los jugadores y el modelo táctico preciso que permita obtener la mejor eficacia y efíciencia posible en el juego (Trninic' et al., 2009). Así que se convierte en necesario saber los apropiados niveles de rendimiento de cada jugador acorde a sus habilidades. En esta búsqueda, los investigadores y el presente trabajo tratan de desarrollar indicadores que ofrezcan un conocimiento más profundo del juego para optimizar la gestión de los equipos.

El análisis del rendimiento del jugador es el que más análisis, literatura e indicadores ha generado, desde el índice TENDEX de Heeren (1992), el ratio de rendimiento-performance index rating utilizado en competiciones de baloncesto europeas, donde básicamente se crea partir de la diferencia entre las acciones positivas del juego (puntos anotados, rebotes, faltas recibidas, sobos de balón y tapones) y las acciones negativas (tiros fallados, faltas cometidas, pérdidas de balón); el Player Efficiency Rating (PER) de Hollinger, o los diferentes índices de valoración más/menos. Ninguno es perfecto, así que tanto desde el ámbito de la investigación como los profesionales del campo tratan de encontrar información a través de nuevos indicadores sencillos de utilizar y que, con los datos disponibles, ofrezcan información inmediata y válida del rendimiento del jugador. 
En esta línea, vemos la posibilidad de profundizar a través de la inclusión del concepto de regularidad. Pese a que algunos indicadores introducen el concepto con la estimación del rendimiento en función de los minutos que juega dicho jugador, enlazándolo con el concepto de eficiencia, creemos que es posible profundizar más en este campo y unir mejor el concepto de rendimiento con regularidad a través de un análisis exhaustivo de las herramientas disponibles existentes a nivel estadístico. En la investigación deportiva encontramos en esta línea el uso de diferentes indicadores, como la entropía (de Saa Guerra et al., 2014; Horowitz, 1997; Sampaio y Maças, 2012), la desviación típica, coeficiente de variación, índice de Gini (Schmidt y Berri, 2001, 2002) o el índice de Herfindahl-Hirschman (Depken, 1999, 2002; Owen et al., 2007), todos ellos interesantes pero que creemos que no explican de forma completa el concepto de regularidad vinculado al rendimiento. Para los autores, la regularidad tiene que ver con la forma con la que los jugadores consiguen su rendimiento durante una temporada, ya que jugadores con índices de rendimiento similares no han tenido por qué tener un mismo comportamiento durante la temporada. El objetivo del artículo es la creación de un índice que mida el rendimiento del jugador, teniendo en cuenta su regularidad y que sea fácil de calcular y usar por una amplia rama del baloncesto profesional.

El trabajo se estructura de la siguiente forma: en la sección 2 se realiza una recopilación de índices usados para medir la regularidad, de forma que añadiéndoles la media aritmética se mida también el rendimiento. En la sección 3 se comparan los índices considerados mostrando la superioridad del formado por la media e inversa del coeficiente de variación (índice propuesto por los autores) sobre el resto. Dicho índice es usado en la sección 4 para clasificar los jugadores que disputaron la Liga ACB en la temporada 2014/2015. Finalmente, en la sección 5 se presentan las principales conclusiones del trabajo.

\section{Rendimiento y la regularidad en el baloncesto.}

Como se ha argumentado en la introducción tomar decisiones exclusivamente a partir del rendimiento o la regularidad de un jugador puede no ser lo más adecuado si no se parten de condiciones adecuadas. Por ejemplo, si se desea comparar la regularidad de dos jugadores en cierto apartado estadístico con el objetivo de decidir un fichaje habría que establecer como prerrequisito que las medias de ambos jugadores sean parecidas. Por tal motivo, en la presente sección se presentan algunos índices usados para medir la regularidad y cómo éstos índices se pueden complementar añadiendo la media aritmética como medida del rendimiento.

\section{Índices de regularidad}

En el presente apartado se recogen los índices más usados (dentro de nuestro conocimiento) para medir la concentración o dispersión de un conjunto de datos. Si bien se pueden aplicar en cualquier escenario, en todos los casos consideraremos que se quiere analizar los datos generados por un jugador de baloncesto en un apartado estadístico concreto.

\section{Entropía}

La entropía, $H$, es una medida de incertidumbre que para los datos generados por un individuo en un apartado estadístico concreto (de Saá et al, 2011, Horowitz, 1997), se calcula a partir de:

$$
H=-\sum_{i=1}^{n} w_{i} \log \left(w_{i}\right)
$$


donde $n$ es el número de partidos jugados y $w_{i}$ es la probabilidad de ocurrencia del evento iésimo, en este caso:

$$
w_{i}=\frac{x_{i}}{\sum_{j=1}^{n} x_{j}}, \quad i=1, \ldots, n,
$$

siendo $x_{i}$ el valor del apartado estadístico analizado en el partido i-ésimo. Se debe verificar que $0<w_{i} \leq 1$ con $i=1, \ldots, n$ y es tal que $\sum_{i=1}^{n} w_{i}=1$.

Adviértase que la concentración mínima (equidistribución o mayor regularidad) se obtiene cuando $w_{i}=\frac{1}{n}$ para todo $i$. En tal caso, el máximo valor de la entropía depende del número de partidos jugados ya que:

$$
H_{\max }=-\sum_{i=1}^{n} \frac{1}{n} \log \left(\frac{1}{n}\right)=\log (n) .
$$

Para evitar la dependencia del número de partidos jugados se considera la medida normalizada $H_{n o r m}=\frac{H}{H_{\max }}$ cuyo valor máximo es el uno.

Por otro lado, la concentración máxima (menor regularidad) se obtiene cuando $w_{i}$ es siempre 0 excepto para un caso en el que es igual a 1 . En tal caso, no es posible obtener la entropía al tener que calcular el logaritmo de cero.

\section{Desviación típica}

La desviación típica $S_{x}$ es una característica estadística que mide la dispersión de los datos con respecto a la medida de posición central conocida como media aritmética (Calot 1988). Para los datos generados por un individuo en un apartado estadístico concreto se calcula a partir de la siguiente expresión:

$$
S_{x}=\sqrt{\frac{1}{n} \sum_{i=1}^{n}\left(x_{i}-\bar{x}\right)^{2}},
$$

donde $n$ es el número de partidos jugados, $x_{i}$ el valor del apartado estadístico analizado en el partido i-ésimo y $\bar{x}$ es la media aritmética de dicho apartado estadístico, esto es, $\bar{x}=\frac{1}{n} \sum_{i=1}^{n} x_{i}$.

El mínimo valor que puede tomar esta característica es el cero, de forma que cuanto más próximo se esté a este valor menor será la dispersión de los datos considerados y, por tanto, mayor será la regularidad.

\section{Coeficiente de variación}

El coeficiente de variación $C V$ es una característica de dispersión adimensional que permite calcular y comparar la homogeneidad de distintos conjuntos de datos (Calot, 1988). Para los datos generados por un individuo en un apartado estadístico concreto se calcula a partir de la siguiente expresión:

$$
C V=\frac{S_{x}}{\bar{x}}
$$

donde $S_{x}$ es la desviación típica y $\bar{x}$ es la media aritmética de dicho apartado estadístico. 
El conjunto de datos es más homogéneo (mayor regularidad) cuanto más pequeño sea su coeficiente de variación.

\section{Índice de Gini}

El índice de Gini $I_{G}$ es una característica estadística de concentración que cuantifica si hay un reparto equitativo (todos reciben lo mismo). Esta medida toma valores entre 0 y 1 , de forma que hay un reparto equitativo (equidistribución o mayor regularidad) si $I_{G}=0 \mathrm{y}$ hay concentración máxima (menor regularidad) si $I_{G}=1$ (Calot, 1988).

Adviértase que este índice sólo se puede calcular cuando los datos analizados son no negativos.

\section{Índice de Herfindahl-Hirschman}

El índice de Herfindahl-Hirschman, HHI, es una medida usada para medir el grado de concentración (Depken, 1999; Owen et al, 2007). Para los datos generados por un individuo en un apartado estadístico concreto se calcula a partir de:

$$
H H I=\sum_{i=1}^{n} w_{i}^{2},
$$

donde $n$ es el número de partidos jugados y $w_{i}$ es la probabilidad de ocurrencia del evento iésimo, en este caso:

$$
w_{i}=\frac{x_{i}}{\sum_{j=1}^{n} x_{j}}, \quad i=1, \ldots, n,
$$

siendo $x_{i}$ el valor del apartado estadístico analizado en el partido i-ésimo. Al igual que para la entropía se verifica que $0 \leq w_{i} \leq 1$ con $i=1, \ldots, n$ y es tal que $\sum_{i=1}^{n} w_{i}=1$. La mínima concentración (equidistribución o mayor regularidad) se obtiene cuando $w_{i}=\frac{1}{n}$ para todo $i$. En tal caso, $H H I_{\min }=\frac{1}{n}$. Por otro lado, la concentración máxima (menor regularidad) se obtiene cuando $w_{i}$ es siempre 0 excepto para un caso en el que es igual a 1. En tal caso, $H H I_{\max }=1$.

Para evitar la dependencia del número de partidos jugados se considera la medida normalizada:

$$
H H I_{n o r m}=\frac{n \cdot H H I-1}{n-1},
$$

que toma valores en el intervalo $[0,1]$.

Inversa del coeficiente de variación

La inversa del coeficiente de variación responde a la siguiente expresión:

$$
C V^{-1}=\frac{\bar{x}}{S_{x}}
$$

donde $S_{x}$ es la desviación típica y $\bar{x}$ es la media aritmética de dicho apartado estadístico. El conjunto de datos es más homogéneo (mayor regularidad) cuanto más grande sea el valor de esta medida. 
Salmerón-Gómez, R., y Gómez-Haro, S. (2016). Ampliando horizontes sobre medición del rendimiento y regularidad en el baloncesto profesional. RICYDE. Revista internacional de ciencias del deporte. 45(12), 234249. http://dx.doi.org/10.5232/ricyde2016.04502

En la siguiente sección se motiva el porqué del uso de esta medida y no del coeficiente de variación.

\section{Índices de rendimiento}

Cuando se analiza el rendimiento de un jugador durante una temporada, independientemente de los índices utilizados, se usa el valor medio del índice o característica (puntos, rebotes, valoración, etc) a analizar como medida de posición central que resuma los valores observados. Sin embargo, esta medida presenta ciertas limitaciones como la sensibilidad que presenta a los valores extremos. Esto plantea una pregunta muy interesante ya que jugadores distintos pueden tener unos mismos valores en cualquier índice que mida su rendimiento pese a que la forma en la que producen esos resultados difiera sustancialmente.

Una forma sencilla de medir la forma de obtener dicho rendimiento es considerar la regularidad con la que se obtienen los valores, por ejemplo, sumando a las medidas anteriores de regularidad la media.

Así, por ejemplo, el índice propuesto en el presente artículo para medir el rendimiento y la regularidad de los datos generados por un individuo en un apartado estadístico concreto es el siguiente:

$$
R R=\bar{x}+\frac{\bar{x}}{S_{x}}=\bar{x}+C V^{-1},
$$

donde $S_{x}$ es la desviación típica y $\bar{x}$ es la media aritmética de dicho apartado estadístico.

Puesto que el coeficiente de variación es una medida adimensional, $R R$ no es interpretable desde el punto de vista de la unidad de medida. Ahora bien:

- Este índice mide el rendimiento de un jugador en un determinado apartado estadístico ya que está formado por la medida de posición central conocida como media aritmética, de forma que a mayor media mayor rendimiento.

- De igual forma mide la regularidad del jugador en un determinado apartado estadístico ya que está formado por la inversa del coeficiente de variación, de forma que a mayor valor de esta cantidad mayor regularidad. Esto se debe a que conforme la desviación típica se acerca al cero, su mínimo valor (regularidad máxima), la inversa del coeficiente de variación aumenta ${ }^{1}$.

- Adviértase que la inversa del coeficiente de variación tiene el mismo signo de la media aritmética, por tanto, un rendimiento negativo supondrá que la parte que mide la regularidad también sea negativa. Es decir, si un jugador con rendimiento negativo es regular será penalizado también por tal motivo.

Por tanto, a mayor valor de $R R$ mayor rendimiento y regularidad.

Como veremos en la siguiente sección, la obtención de esta medida como la agregación de las dos características señaladas responde a la intención de salvar limitaciones observadas en las características presentadas anteriormente.

\footnotetext{
${ }^{1}$ Adviértase que si la desviación típica es cero, esta medida quedaría indeterminada. Incluso un valor de la desviación típica extremadamente cercano al cero proporcionaría un valor anormalmente alto para el índice en cuestión. En este caso, estos indicios constituyen una alerta para que dicho caso sea analizado particularmente.
} 
Salmerón-Gómez, R., y Gómez-Haro, S. (2016). Ampliando horizontes sobre medición del rendimiento y regularidad en el baloncesto profesional. RICYDE. Revista internacional de ciencias del deporte. 45(12), 234249. http://dx.doi.org/10.5232/ricyde2016.04502

\section{Comparación de los índices de rendimiento y regularidad}

El juego del baloncesto genera muchísima información numérica, de ahí el gran y diverso número de índices de valoración de jugadores, por encima incluso de los 200 índices (Martínez, 2010a,b,c). Estos índices se clasifican en función de: a) si miden producción defensiva, ofensiva o ambas y b) si son obtenidos a partir del boxscore o del play by play, variando el nivel de complejidad para su cálculo.

Puesto que gran parte de estas características estadísticas a analizar pueden tomar valores negativos, los índices de entropía, de Gini y Herfindahl-Hirschman no pueden ser calculados. Esta cuestión es una importante limitación ya que sólo pueden usarse ${ }^{2}$ en las características estadísticas mostradas en el boxscore. Seguramente por este motivo su aplicación ha sido en el análisis de si las competiciones son o no competitivamente equilibradas (de Saá et al, 2011; Depken, 1999; Horowitz 1997; Owen et al, 2007) ya que en tal caso se trabaja a partir del número de victorias obtenidas por cada equipo que son datos positivos.

Para comparar los restantes índices se consideran los siguientes datos obtenidos por tres jugadores hipotéticos en un determinado apartado estadístico:

$$
\begin{gathered}
\text { Jugador } 1 \text { (J1): 12, 8, 12, 8, } 10 \\
\text { Jugador } 2 \text { (J2): 6, 4, 20, 6, } 20 \\
\text { Jugador } 3 \text { (J3): 2, 3, 2, 3, } 4
\end{gathered}
$$

En la Tabla 1 se obtienen los resultados de los índices de regularidad y rendimiento para los tres casos anteriores. Si tenemos en cuenta la media, el segundo jugador es el de mayor rendimiento, mientras que a partir de la desviación típica el más regular es el tercero y a partir del coeficiente de variación sería preferible el primero. Es más, el jugador menos regular es el segundo que es el que tiene mayor rendimiento. ¿Qué jugador es preferible? Parece claro que tomar una decisión exclusivamente a partir de una de las dos dimensiones no es lo más idóneo.

Tabla 1. Valores de los índices de regularidad y rendimiento para datos inventados

\begin{tabular}{r|c|c|c}
\multicolumn{1}{c|}{ Índice } & $\mathbf{J 1}$ & $\mathbf{J 2}$ & $\mathbf{J 3}$ \\
\hline Media & 10 & 11.2 & 2.8 \\
\hline Desviación Típica & 2 & 8.074 & 0.836 \\
\hline Coeficiente de Variación & 0.2 & 0.721 & 0.298 \\
\hline Media + Desviación Típica & 12 & 19.274 & 3.636 \\
\hline Rendimiento-Regularidad (RR) & 15 & 12.587 & 6.146 \\
& & &
\end{tabular}

\footnotetext{
${ }^{2}$ Realmente la entropía tampoco podría calcularse en estos casos, ya que no puede obtenerse si en los datos con los que se trabaja aparece el cero.
} 
Salmerón-Gómez, R., y Gómez-Haro, S. (2016). Ampliando horizontes sobre medición del rendimiento y regularidad en el baloncesto profesional. RICYDE. Revista internacional de ciencias del deporte. 45(12), 234249. http://dx.doi.org/10.5232/ricyde2016.04502

Considerando añadir a las medidas de regularidad la media aritmética se tiene que:

- Sumar la media a la desviación típica no tiene sentido ya que el índice resultante no se podría interpretar: mayor media supone mayor rendimiento pero mayor desviación típica implica menos regularidad.

- Exactamente lo mismo ocurre al añadir la media al coeficiente de variación.

- Sin embargo, tal y como se ha comentado, un mayor valor de $R R$ si está asociado a un mayor rendimiento y una mayor regularidad.

Por tanto, el índice Rendimiento-Regularidad $(R R)$ parece que muestra un mejor comportamiento $^{3}$ a la hora de medir el rendimiento y regularidad de un jugador en un determinado apartado estadístico. Siguiendo este índice, el jugador preferible sería el primero.

\section{Resultados}

Una vez justificada la elección del índice $R R$, en la presente sección se especifica el apartado estadístico que se va a usar para calcularlo en los 193 jugadores que han disputado al menos 10 partidos en la Liga ACB durante la temporada 2014/2015 con una media de minutos jugados superior o igual a 10. Este límite obedece a una necesidad de los autores de trabajar con unos datos de jugadores que hayan participado de forma constante en la temporada para la legitimidad de los indicadores propuestos en el trabajo (Berri, Brook y Schmidt, 2007; Berri y Krautmann, 2006, Cooper, Ruiz y Sirvent, 2009).

Puesto que la media aritmética y la desviación típica son sensibles a los datos extremos se tiene que $R R$ presenta este mismo problema. Es claro que precisamente estos datos extremos son la base del concepto de regularidad presentado en el trabajo, por lo que se hace necesario distinguir entre datos altos y bajos y datos anormalmente altos y bajos. Sin duda, los segundos distorsionarían el análisis realizado. Dentro de la estadística descriptiva existen técnicas que permiten detectar la existencia de este tipo de datos, sin embargo, siguiendo la premisa de que los índices propuestos deben ser fáciles de calcular y entendiendo que un análisis de este tipo de la muestra no lo facilitaría se ha optado por descartar el $5 \%$ de los datos más grandes y más pequeños. Realizar estos cálculos eliminando esta información de la muestra es fácil usando los paquetes informáticos disponibles a día de hoy.

\section{Apartado estadístico analizado}

En este caso se ha optado por calcular el rendimiento y la regularidad en una medida que proporcione una visión global de la actuación de un jugador en un partido obviando su anotación. En tal caso, se transformará de forma conveniente la Valoración $A C B^{5}$, que es el apartado estadístico usado en la Liga ACB para cuantificar el comportamiento de un jugador en un determinado partido. Más concretamente, se trabajará con la siguiente transformación de este índice:

\footnotetext{
${ }^{3}$ Sería interesante en posteriores trabajos realizar simulaciones de datos para confirmar esta prevalencia y comprobar la estabilidad de $R R$ ante distintos escenarios.

${ }^{4}$ Estos trabajos establecen límites mayores, 40 partidos jugados y 12 minutos de media. Hay que tener en cuenta que la NBA es una competición de 82 partidos (la ACB, 38) y 48 minutos partido (40 en ACB).

${ }^{5}$ Valoración $\mathrm{ACB}=$ Puntos anotados + Rebotes + Asistencias + Recuperaciones + Faltas personales recibidas + Tapones realizados - Tiros fallados - Pérdidas - Tapones recibidos - Faltas cometidas.
} 
Salmerón-Gómez, R., y Gómez-Haro, S. (2016). Ampliando horizontes sobre medición del rendimiento y regularidad en el baloncesto profesional. RICYDE. Revista internacional de ciencias del deporte. 45(12), 234249. http://dx.doi.org/10.5232/ricyde2016.04502

$$
\text { ValPtosMin }=\frac{\text { Valoración ACB }- \text { Puntos anotados }}{\text { Minutos jugados }}=\frac{\text { Resto }}{\text { Minutos jugados }},
$$

siendo Resto $=$ Rebotes + Asistencias + Recuperaciones + Faltas personales recibidas + Tapones realizados - Tiros fallados - Pérdidas - Tapones recibidos - Faltas cometidas.

De esta forma se elimina la dependencia que tiene la Valoración ACB de los puntos anotados y el posible efecto acumulación debido a jugar un mayor número de minutos. Esta medida es parecida al TENDEX ${ }^{6}$, índice muy extendido para evaluar el comportamiento de un jugador.

Además es posible una nueva interpretación:

- Si ValPtosMin $>0$ entonces Resto $>0$, es decir, el número de acciones positivas realizadas por el jugador supera a las negativas.

- Si ValPtosMin $<0$ entonces Resto < 0 , es decir, el número de acciones negativas realizadas por el jugador supera a las positivas.

Por tanto, obviando el perfil anotador (el cual puede obtenerse de forma inmediata a partir de los puntos anotados), este índice podría usarse para detectar qué jugadores son más completos.

\section{Aplicación a la Liga Española de Baloncesto- $A C B$}

En esta subsección se van a clasificar jugadores de la Liga ACB de la temporada 2014/15 a partir de su rendimiento y regularidad en el apartado estadístico ValPtosMin.

En primer lugar, teniendo en cuenta las representaciones de los Gráficos 1 y 2, a partir de los datos de la Tabla 2 (en el apéndice) se observa que:

- Es clara la relación de a mayor media mayor valor de $R R$. El coeficiente de correlación entre ambas medidas es de 0.9791 (significativamente distinto de cero al 5\% ya que el p-valor asociado es menor que $10^{-3} \mathrm{y}$, por tanto, menor que 0.05 ).

- Sin embargo, en un principio la relación de a menor dispersión mayor valor de $R R$ no se verifica. El coeficiente de correlación entre ambas medidas es de -0.1554 (no significativamente distinto de cero al $5 \%$ ya que el p-valor, 0.1258 , es mayor que 0.05 ). Esto se puede deber a que los jugadores con valores negativos en RR lo hacen con regularidad y el signo negativo impide que el coeficiente de correlación capte de forma adecuada la relación. Si se considera el valor absoluto de $R R$, su correlación con la desviación típica es de -0.1857 , siendo en este caso significativamente distinta de cero al $5 \%$ (su p-valor, 0.0097, es menor que 0.05). Esta correlación indicaría que a menor desviación típica mayor valor de $R R$.

- $\quad$ El jugador más valorado en la liga regular, Andy Panko (AP7, Fuenlabrada), también es el máximo anotador y el jugador que más minutos ha disputado (ver ACB Noticias Digital número 936). Si se elimina la influencia de los puntos y minutos en la valoración este jugador pasa a ocupar la posición 81 . Esto podría justificar su no inclusión dentro del mejor quinteto de la Liga ACB.

\footnotetext{
${ }^{6}$ TENDEX $=($ Puntos + Rebotes + Asistencias + Recuperaciones + Tapones - Tiros de campo fallados $-0.5 *$ Tiros libres fallados - Pérdidas - Faltas cometidas)/Minutos.
} 
- $\quad$ El jugador elegido como MVP Orange 2014/15, Felipe Reyes (FR9, Real Madrid), ocupa el puesto 17 al eliminar la influencia de los puntos y minutos jugados. Este jugador también es integrante del mejor quinteto de la liga.

- En los primeros 8 puestos del ranking establecido se tiene que 6 de ellos son jugadores interiores: Marko Todorovic (MT11, Bilbao, posición 1), Augusto Lima (AL23, Murcia, posición 2), Walter Tavares (WT22, Gran Canaria, posición 4), Ante Tomic (AT44, Barcelona, posición 6), Fran Vázquez (FV17, Unicaja, posición 7) y Mirza Begic (MB15, Baskonia, posición 8).

- Por tanto, se podría pensar que ValPtosMin favorece a jugadores con perfil interior, teniendo mayor mérito las posiciones ocupadas por Jared Jordan (JJ10, Gipuzkoa, base, puesto 5), Tomas Satoransky (TS13, Barcelona, base, puesto 9) o Pau Ribas (PR5, Valencia, escolta, puesto 10).

- Considerando el ranking establecido el jugador mejor situado es Marko Todorovic (MT11, Bilbao), seguido por Augusto Lima (AL23, Murcia). Por lo que, siguiendo este apartado estadístico, se podrían considerar como los mejores jugadores de la temporada 2014/15 en la Liga ACB.

Finalmente, con el objetivo de analizar si el número de encuentros o minutos disputados influye en el comportamiento del jugador en el índice $R R$ se calcula en primer lugar el diagrama de dispersión de estas variables atisbándose una relación positiva (ver Gráficos 3 y 4 del apéndice). Esta relación se confirma al calcular el coeficiente de correlación entre dichas medidas puesto que se obtienen, respectivamente, valores iguales a 0.334 y 0.2606 (significativamente distintos de cero al 5\% ya que sus p-valores asociados son menores que $10^{-3} \mathrm{y}$, por tanto, menores que 0.05 ). Por tanto, a mayor número de partidos y minutos disputados mayor valor de $R R$.

\section{Conclusiones}

Las personas que toman las decisiones en los equipos deportivos, directores técnicos y entrenadores, necesitan disponer de la mejor información posible para poder tomar decisiones correctas. Actualmente, los investigadores tratan de desarrollar instrumentos de medición con el que poder evaluar el comportamiento de los jugadores durante el juego. Es éste el objetivo del presente trabajo, desarrollar un índice sencillo de calcular que mida el rendimiento de un jugador premiando al mismo tiempo su regularidad.

Con tal objetivo, se ha realizado una recopilación de medidas usadas para medir la regularidad, las cuales consisten en medir de alguna forma la mayor o menor equidistribución. Tras añadir a estas medidas la media aritmética (forma habitual de resumir un conjunto de datos y considerándola como un buen indicador del rendimiento del jugador), se ha procedido a compararlas con la medida propuesta. De esta forma se ha visto la prevalencia de ésta última sobre el resto, aunque recordemos una vez más que sería interesante realizar simulaciones de datos para confirmar esta superioridad y comprobar la estabilidad de $R R$ ante distintos escenarios.

Debido a la sensibilidad de la media y desviación típica a los datos extremos, las conclusiones tomadas a partir del índice presentado podrían ser erróneas. Para evitar estas posibles incongruencias se ha optado por eliminar antes de realizar el análisis el 5\% de los datos más grandes y más pequeños. En una competición donde se disputan entre 35 y 40 partidos por temporada, como es la Liga ACB, esto supone descartar los dos encuentros con peor y mejor comportamiento del jugador. 
Salmerón-Gómez, R., y Gómez-Haro, S. (2016). Ampliando horizontes sobre medición del rendimiento y regularidad en el baloncesto profesional. RICYDE. Revista internacional de ciencias del deporte. 45(12), 234249. http://dx.doi.org/10.5232/ricyde2016.04502

En este punto y en relación con las simulaciones comentadas, podría ser interesante estudiar el comportamiento del índice en situaciones donde hay datos extremos y analizar, por ejemplo, las diferencias existentes cuando se calcula el índice con toda la muestra y la muestra truncada.

Al mismo tiempo, el concepto de anotación está sobreevaluado en el baloncesto (Berri, Brook, y Schmidt, 2007), siendo el factor de más peso en índices muy empleados a nivel profesional como TENDEX. Por tal motivo, para aplicar el índice presentado se ha considerado un índice de rendimiento que no sea tan dependiente del concepto de anotación y del tiempo que el jugador permanece en el campo.

Finalmente, se ha analizado la relación existente con el número de partidos jugados y el número de minutos disputados, obteniéndose una relación directa. Ahora bien, hay que tener en cuenta que los datos usados se limitan a los partidos disputados durante la Liga ACB y muchos de los jugadores considerados juegan alguna competición europea (con el efecto que pueda tener sobre su comportamiento) y este factor no se ha tenido en cuenta en este análisis.

\section{Referencias}

ACB Noticias Digital número 936. Disponible en internet en la dirección web: http://www.acb.com/pdfs/num936.pdf.

Alamar, B. C. (2013). Sports analytics: A guide for coaches, managers, and other decision makers. Columbia University Press. http://dx.doi.org/10.7312/columbia/9780231162920.001.0001

Berri, D. J.; Brook, S. L., \& Schmidt, M. B. (2007). Does One Simply Need to Score to Score? International Journal of Sport Finance, 2, 190-205. http://dx.doi.org/10.1093/ei/cbj033

Berri, D. J. y Krautmann, A. C. (2006). Shrinking on the court: testing for the incentives effects of guaranteed pay. Economic Inquiry, 44(3), 536-546.

Calot, G. (1988). Curso de Estadística Descriptiva. Paraninfo, S.A. Madrid.

Cooper, W. W., Ruiz, J. L., y Sirvent, I. (2009). Selecting non-zero weights to evaluate effectiveness of basketball players with DEA. European Journal of Operational Research, 195, 563-574. http://dx.doi.org/10.1016/j.ejor.2008.02.012

Depken, C.A. (1999). Free-agency and the competitiveness of major league baseball. Review of Industrial Organization, 14, 205-217. http://dx.doi.org/10.1023/A:1007788303098

Depken, C. A. (2002). Free agency and the concentration of player talent in Major League Baseball, Journal of Sports Economics, 3, 335-353. http://dx.doi.org/10.1177/152700250200300403

De Saá, Y.; Martín, J. M.; Arjonilla, N.; Sarmiento, S.; Rodríguez, D. y García, J. M. (2011). Analysis of competitiveness in the NBA regular seasons. Ugdymas Küno Kultüra Sportas, 1 (80), 17-21.

De Saa Guerra, Y., Martín-González, J.M. y García Manso, J.M. (2014). El baloncesto desde la perspectiva de los sistemas complejos no-lineales. Revista Española de Educación Física y Deportes, 406, 45-55.

Drust, B. (2010). Performance analysis research: Meeting the challenge. Journal of Sports Sciences, 28(9), 921-922. http://dx.doi.org/10.1080/02640411003740769 
Salmerón-Gómez, R., y Gómez-Haro, S. (2016). Ampliando horizontes sobre medición del rendimiento y regularidad en el baloncesto profesional. RICYDE. Revista internacional de ciencias del deporte. 45(12), 234249. http://dx.doi.org/10.5232/ricyde2016.04502

Gutierrez, O., \& Ruiz, J. L. (2013). Data envelopment analysis and cross-efficency evaluation in the management of sport teams: the assessment of game performance of players in the Spanish handball league. Journal of Sport Management, 27(3), 217229.

Heeren, D. (1992). Basketball Abstract, 1991-92 Edition. New Jersey: Prentice Hall.

Horowitz, I. (1997). The increasing competitive balance in major league baseball. Review of industrial Organization, 12, 373-387. http://dx.doi.org/10.1023/A:1007799730191

Lewis, M. (2004). Moneyball. New York: Norton.

Martínez, J. A. (2010a). Una revisión de los sistemas de valoración de jugadores (I). Descripción de los métodos existentes. Revista Internacional de Derecho y Gestión del Deporte, 10, 37-78.

Martínez, J. A. (2010b). Una revisión de los sistemas de valoración de jugadores de baloncesto (II). Competiciones oficiales y ligas de fantasía. Revista Internacional de Derecho y Gestión del Deporte, 11, 48-68.

Martínez, J. A. (2010c). Una revisión de los sistemas de valoración de jugadores de baloncesto (III). Discusión general. Revista Internacional de Derecho y Gestión del Deporte, 12, 44-79.

Owen, P.D.; Ryan, M. \& Weatherston, C.R. (2007). Measuring competitive balance in professional team sports using the Herfindahl-Hirschman index. Review of Industrial Organization, 31, 289-302. http://dx.doi.org/10.1007/s11151-008-9157-0

Sampaio, J. \& Maças, V. (2012). Measuring Tactical Behaviour in Football. International Journal of Sports Medicine, 33, 395-401. http://dx.doi.org/10.1055/s-0031-1301320

Schmidt, M. B., \& Berri, D.J. (2001). Competition and Attendance: The Case of Major League Baseball, Journal of Sports Economics, 2, 147-167. http://dx.doi.org/10.1177/152700250100200204

Schmidt, M.B., \& Berri, D.J. (2002). Competitive balance and market size in Major League Baseball: A response to Baseball's Blue Ribbon panel, Review of Industrial Organization, 21, 41-54. http://dx.doi.org/10.1023/A:1016045011186

Trninic', M.; Trninic', S., \& Papic', V. (2009). Development Management Model of Elite Athletes in Team Sports Games. Collegium Antropologicum, 33(2), 363-372.

\section{Agradecimientos}

Los autores desean agradecer al departamento de Métodos Cuantitativos para la Economía y la Empresa de la Universidad de Granada la provisión de fondos para la investigación. También agradecemos a los revisores anónimos sus sugerencias y comentarios en el proceso de evaluación. También agradecer al departamento de Organización de empresas y a los proyectos estatales ECO2013-47009-P y autonómicos P11-SEJ-7988. 


\section{Anexos}

Tabla 2. Clasificación a partir del cálculo de $R R$ para ValPtosMin de los 193 jugadores estudiados

\begin{tabular}{|c|c|c|c|c|c|}
\hline Posición & Jugador & $\mathbf{R R}$ & Posición & Jugador & RR \\
\hline 1 & MT11 & 2.2193 & 97 & AN6 & -0.0862 \\
\hline 2 & AL23 & 2.1418 & 98 & CB6 & -0.1028 \\
\hline 3 & CS12 & 1.9301 & 99 & NR4 & -0.1050 \\
\hline 4 & WT22 & 1.7012 & 100 & BN8 & -0.1281 \\
\hline 5 & JJ10 & 1.6110 & 101 & AU3 & -0.1659 \\
\hline 6 & AT44 & 1.5670 & 102 & RM17 & -0.1693 \\
\hline 7 & FV17 & 1.5148 & 103 & GL12 & -0.1791 \\
\hline 8 & MB15 & 1.5061 & 104 & RS10 & -0.1953 \\
\hline 9 & TS13 & 1.4692 & 105 & MG13 & -0.2019 \\
\hline 10 & PR5 & 1.4318 & 106 & MK19 & -0.2036 \\
\hline 11 & RU6 & 1.3835 & 107 & RN8 & -0.2227 \\
\hline 12 & LS43 & 1.3411 & 108 & AM31 & -0.2471 \\
\hline 13 & JB33 & 1.3286 & 109 & DC10 & -0.2625 \\
\hline 14 & KI55 & 1.2110 & 110 & SB4 & -0.2706 \\
\hline 15 & OB12 & 1.2052 & 111 & JF7 & -0.2708 \\
\hline 16 & LW21 & 1.2036 & 112 & DD13 & -0.3041 \\
\hline 17 & FR9 & 1.0815 & 113 & SL12 & -0.3067 \\
\hline 18 & PL12 & 1.0471 & 114 & ML24 & -0.3421 \\
\hline 19 & sV6 & 1.0342 & 115 & JV9 & -0.3430 \\
\hline 20 & QC11 & 1.0100 & 116 & NJ32 & -0.3603 \\
\hline 21 & HN25 & 0.9735 & 117 & JF30 & -0.3674 \\
\hline 22 & SL23 & 0.9452 & 118 & AM10 & -0.3842 \\
\hline 23 & TH22 & 0.9143 & 119 & DB1 & -0.3850 \\
\hline 24 & SS17 & 0.9111 & 120 & LB19 & -0.3945 \\
\hline 25 & GA14 & 0.8786 & 121 & JCN11 & -0.3980 \\
\hline 26 & JT88 & 0.7658 & 122 & HG31 & -0.4098 \\
\hline 27 & WH11 & 0.7344 & 123 & KL25 & -0.4169 \\
\hline 28 & JG15 & 0.7279 & 124 & NR11 & -0.4199 \\
\hline 29 & FC5 & 0.7099 & 125 & JA6 & -0.4259 \\
\hline 30 & JA17 & 0.7016 & 126 & JR20 & -0.4266 \\
\hline 31 & $\mathrm{Cl} 4$ & 0.6928 & 127 & CG30 & -0.4324 \\
\hline 32 & ZS3 & 0.6889 & 128 & IF42 & -0.4482 \\
\hline 33 & RF5 & 0.6844 & 129 & BG11 & -0.4490 \\
\hline 34 & MH9 & 0.6834 & 130 & JS14 & -0.4737 \\
\hline 35 & MS44 & 0.6620 & 131 & MH8 & -0.5264 \\
\hline 36 & NM11 & 0.6472 & 132 & P018 & -0.5293 \\
\hline 37 & DS20 & 0.6270 & 133 & MJ3 & -0.5421 \\
\hline 38 & TB21 & 0.6255 & 134 & SR27 & -0.5519 \\
\hline 39 & MK14 & 0.6243 & 135 & MG16 & -0.5696 \\
\hline 40 & HR19 & 0.6121 & 136 & RG44 & -0.5705 \\
\hline 41 & VG32 & 0.6031 & 137 & GB13 & -0.5744 \\
\hline 42 & WH14 & 0.6029 & 138 & KC4 & -0.5998 \\
\hline 43 & EB13 & 0.6013 & 139 & EJ25 & -0.6006 \\
\hline 44 & DD33 & 0.5929 & 140 & BD14 & -0.6058 \\
\hline 45 & RL55 & 0.5882 & 141 & SS9 & -0.6392 \\
\hline 46 & PA34 & 0.5770 & 142 & TK22 & -0.6530 \\
\hline 47 & AH17 & 0.5504 & 143 & $\mathrm{CH} 11$ & -0.6586 \\
\hline 48 & LH44 & 0.5445 & 144 & RK14 & -0.6679 \\
\hline
\end{tabular}




\begin{tabular}{|c|c|c|c|c|c|}
\hline 49 & RSMO & 0.5298 & 145 & AA16 & -0.6740 \\
\hline 50 & CC10 & 0.5180 & 146 & SB8 & -0.6781 \\
\hline 51 & JT8 & 0.5083 & 147 & NK52 & -0.6786 \\
\hline 52 & MD12 & 0.5020 & 148 & NN8 & -0.6853 \\
\hline 53 & PP6 & 0.5013 & 149 & RL31 & -0.6873 \\
\hline 54 & SJ89 & 0.4957 & 150 & AB24 & -0.6954 \\
\hline 55 & TB11 & 0.4362 & 151 & AA10 & -0.6963 \\
\hline 56 & VL13 & 0.4327 & 152 & DST23 & -0.7111 \\
\hline 57 & JS15 & 0.4133 & 153 & LR34 & -0.7132 \\
\hline 58 & DN15 & 0.3981 & 154 & KV7 & -0.7240 \\
\hline 59 & VS7 & 0.3794 & 155 & JG22 & -0.7247 \\
\hline 60 & IB30 & 0.3735 & 156 & NR5 & -0.7254 \\
\hline 61 & DP5 & 0.3711 & 157 & DW34 & -0.7693 \\
\hline 62 & JH41 & 0.3696 & 158 & DH45 & -0.7953 \\
\hline 63 & JD5 & 0.3656 & 159 & DB42 & -0.7957 \\
\hline 64 & AD4 & 0.3386 & 160 & AS33 & -0.8114 \\
\hline 65 & SVR9 & 0.3355 & 161 & BN34 & -0.8890 \\
\hline 66 & SR13 & 0.3225 & 162 & MB31 & -0.8925 \\
\hline 67 & NL9 & 0.2636 & 163 & KP8 & -0.8978 \\
\hline 68 & TS5 & 0.2481 & 164 & BH23 & -0.9133 \\
\hline 69 & WT10 & 0.2445 & 165 & AW21 & -0.9289 \\
\hline 70 & FSE19 & 0.2227 & 166 & RT9 & -0.9579 \\
\hline 71 & PT19 & 0.1873 & 167 & RH5 & -0.9588 \\
\hline 72 & GV16 & 0.1838 & 168 & AR8 & -0.9626 \\
\hline 73 & ML30 & 0.1697 & 169 & DT20 & -1.0017 \\
\hline 74 & $\mathrm{AH} 11$ & 0.1588 & 170 & JC20 & -1.0075 \\
\hline 75 & AV14 & 0.1491 & 171 & TU10 & -1.0374 \\
\hline 76 & BW10 & 0.1448 & 172 & SG21 & -1.0572 \\
\hline 77 & KT14 & 0.1430 & 173 & DJ35 & -1.0671 \\
\hline 78 & TK21 & 0.1135 & 174 & AM15 & -1.0875 \\
\hline 79 & FL19 & 0.1119 & 175 & DMO & -1.0878 \\
\hline 80 & TS7 & 0.1012 & 176 & JS20 & -1.1427 \\
\hline 81 & AP7 & 0.0990 & 177 & VS19 & -1.1590 \\
\hline 82 & 1025 & 0.0912 & 178 & SV18 & -1.2142 \\
\hline 83 & DM5 & 0.0812 & 179 & BC20 & -1.2411 \\
\hline 84 & AO4 & 0.0803 & 180 & AC33 & -1.3346 \\
\hline 85 & BR41 & 0.0699 & 181 & KK24 & -1.3647 \\
\hline 86 & PP44 & 0.0347 & 182 & CG43 & -1.3953 \\
\hline 87 & DP8 & 0.0299 & 183 & DB9 & -1.4805 \\
\hline 88 & FVL4 & 0.0177 & 184 & II20 & -1.6065 \\
\hline 89 & KP6 & 0.0154 & 185 & RM10 & -1.6189 \\
\hline 90 & BO24 & 0.0057 & 186 & DA33 & -1.6241 \\
\hline 91 & SM4 & -0.0049 & 187 & JB20 & -1.6995 \\
\hline 92 & KL10 & -0.0216 & 188 & BD12 & -1.7004 \\
\hline 93 & BS9 & -0.0582 & 189 & EW34 & -1.7283 \\
\hline 94 & LK14 & -0.0670 & 190 & DA1 & -1.8603 \\
\hline 95 & JM8 & -0.0749 & 191 & JM1 & -2.1546 \\
\hline \multirow[t]{2}{*}{96} & GS12 & -0.0770 & 192 & SW15 & -2.1749 \\
\hline & & & 193 & SB15 & -2.7845 \\
\hline
\end{tabular}


Salmerón-Gómez, R., y Gómez-Haro, S. (2016). Ampliando horizontes sobre medición del rendimiento y regularidad en el baloncesto profesional. RICYDE. Revista internacional de ciencias del deporte. 45(12), 234249. http://dx.doi.org/10.5232/ricyde2016.04502

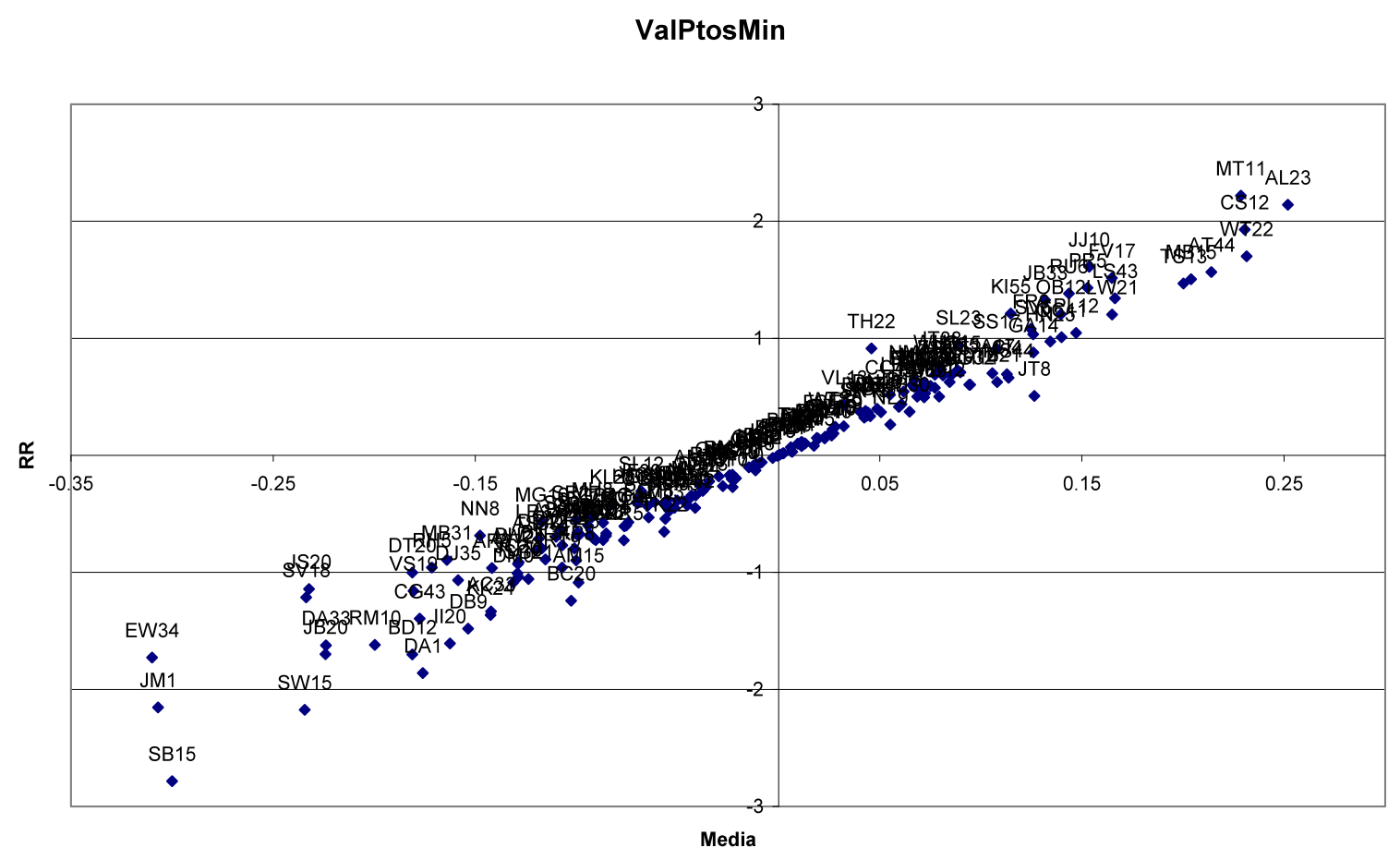

Gráfico 1. Diagrama de dispersión de $R R$ frente a la media para ValPtosMin

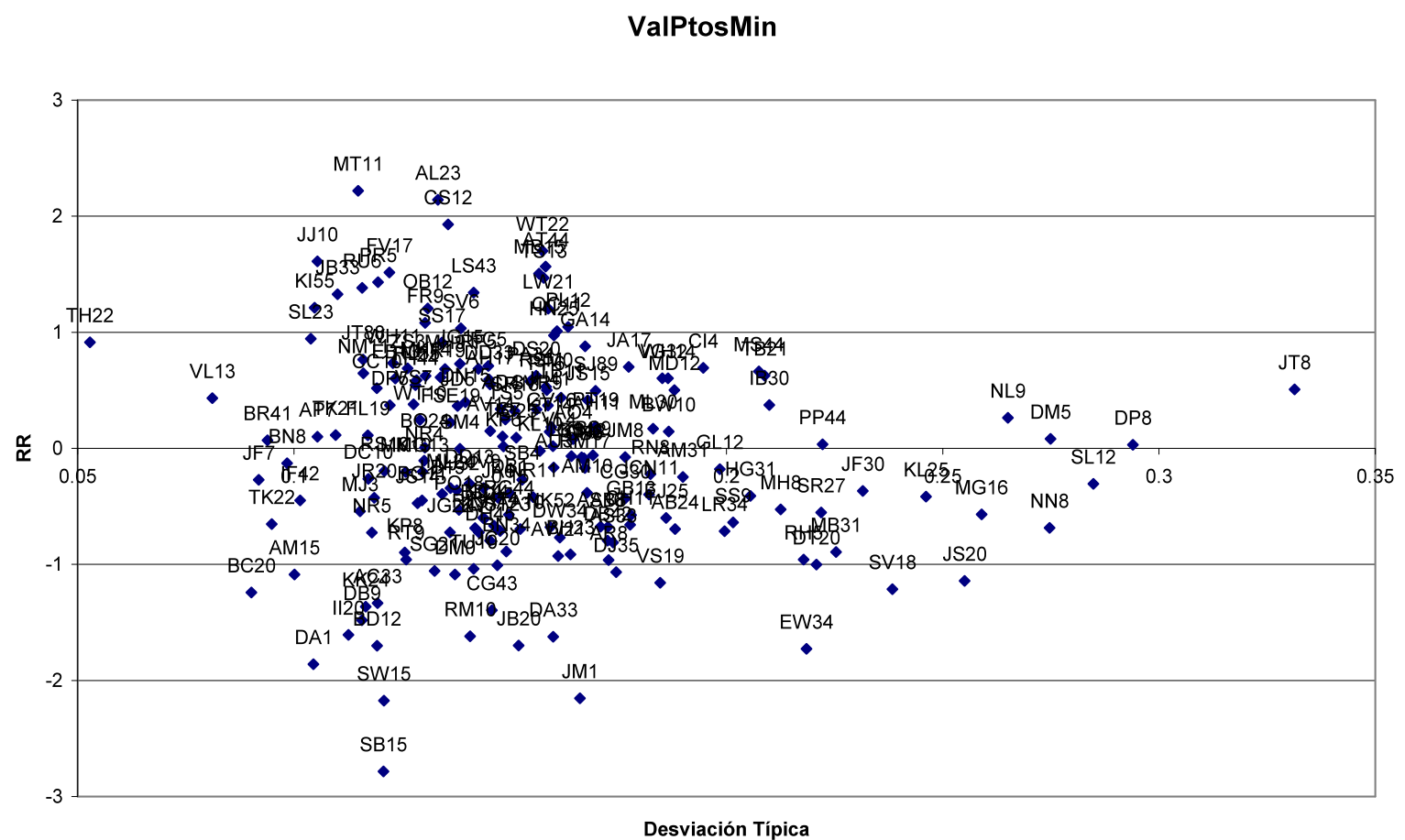

Gráfico 2. Diagrama de dispersión de $R R$ frente a la desviación típica para ValPtosMin 
Salmerón-Gómez, R., y Gómez-Haro, S. (2016). Ampliando horizontes sobre medición del rendimiento y regularidad en el baloncesto profesional. RICYDE. Revista internacional de ciencias del deporte. 45(12), 234249. http://dx.doi.org/10.5232/ricyde2016.04502

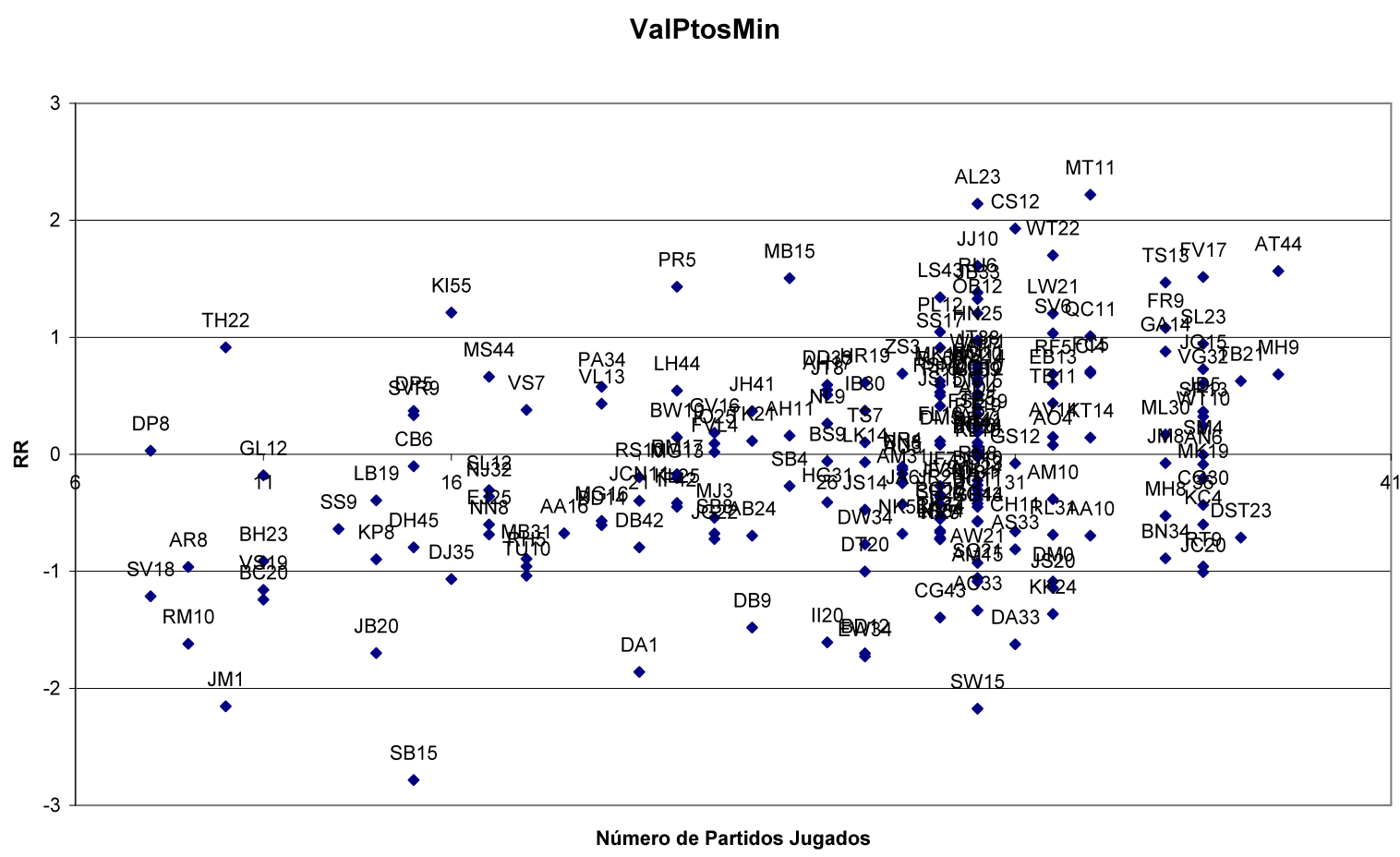

Gráfico 3. Diagrama de dispersión de $R R$ frente al número de partidos jugados para ValPtosMin

\section{ValPtosMin}

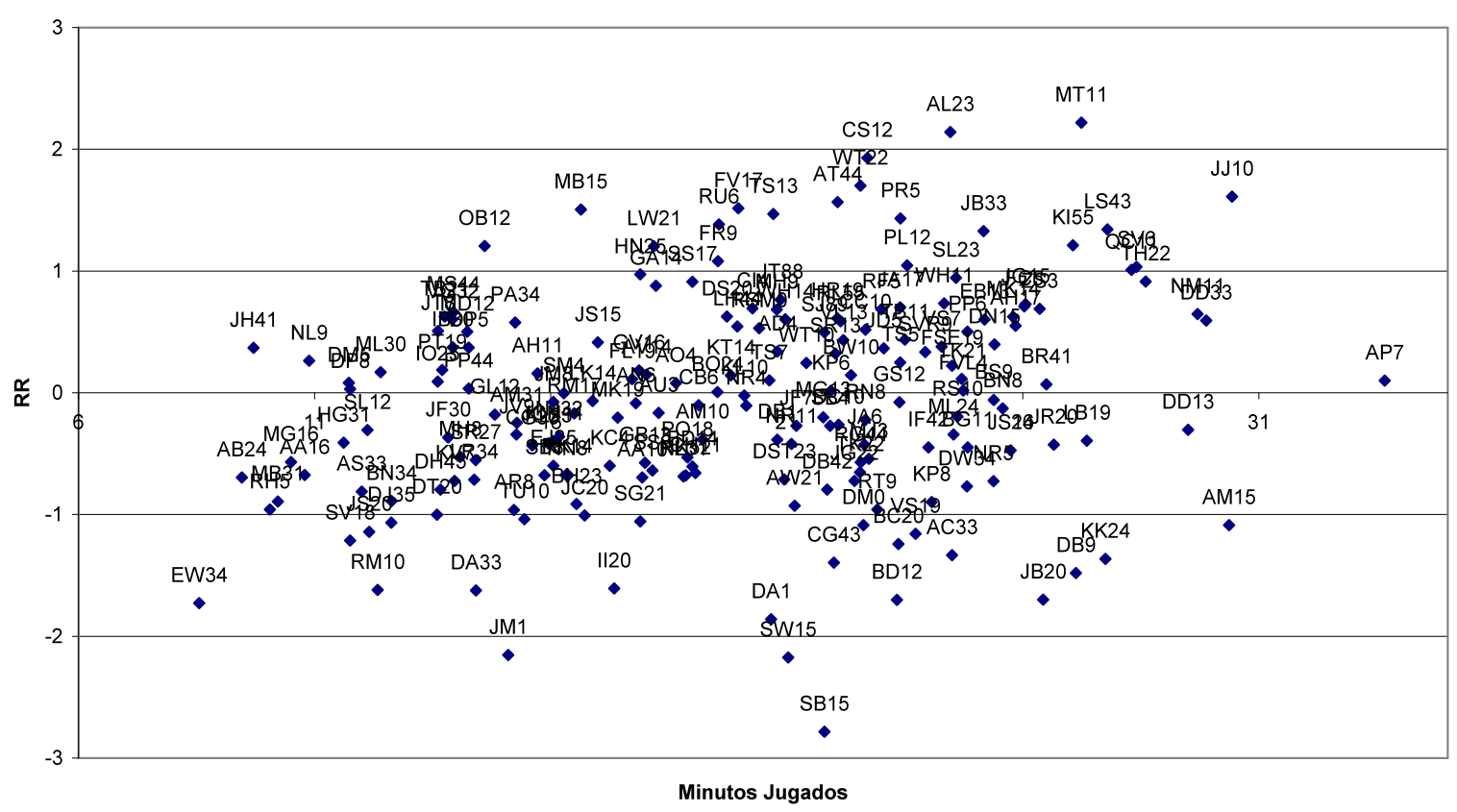

Gráfico 4. Diagrama de dispersión de $R R$ frente al número de minutos jugados para ValPtosMin 